\title{
Correlation analysis of muscle amino acid deposition and gut microbiota profile of broilers reared at different ambient temperatures
}

\author{
Yuting Yang ${ }^{1, \mathrm{a}}$, Huan $\mathrm{Gao}^{1, \mathrm{a}}$, Xing $\mathrm{Li}^{1}$, Zhenhui Cao ${ }^{1}$, Meiquan $\mathrm{Li}^{2}$, Jianping $\mathrm{Liu}^{3}$, \\ Yingying Qiao ${ }^{1}$, Li Ma ${ }^{4}$, Zhiyong Zhao ${ }^{5}$, and Hongbin Pan ${ }^{1}$,
}

\author{
* Corresponding Author: Hongbin Pan \\ Tel: +86-0871-65227798, \\ Fax: +86-0871-65227798 \\ E-mail:ynsdyz@163.com
}

${ }^{1}$ Yunnan Provincial Key Laboratory of Animal Nutrition and Feed Science, Faculty of

Animal Science and Technology, Yunnan Agricultural University, Kunming 650201 China

2 Department of Animal Husbandry and Veterinary Medicine, College of Agriculture, Kunming University, Kunming 650201, China Jiangsu Key Laboratory for Molecular and Medical Biotechnology, College of Life Sciences, Nanjing Normal University, Nanjing 210023, China

${ }^{4}$ Yunnan Vocational and Technical College of Agriculture, Kunming 650201, China

${ }^{5}$ Yunnan Animal Science and Veterinary Institute, Kunming 650201, China

a Both authors contribute equally to this work

ORCID

Yuting Yang

https://orcid.org/0000-0002-4781-1068

Huan Gao

https://orcid.org/0000-0002-9700-4547

Xing Li

https://orcid.org/0000-0003-4012-4123

Zhenhui Cao

https://orcid.org/0000-0002-5344-134X

Meiquan Li

https://orcid org/0000-0003-0292-6328

Jianping Liu

https://orcid.org/0000-0002-7336-3895

Yingying Qiao

https://orcid.org/0000-0002-0090-6430 Li Ma

https://orcid.org/0000-0003-4434-2363

Zhiyong Zhao

https://orcid.org/0000-0003-1186-5256

Hongbin Pan

https://orcid.org/0000-0002-9289-2434

Submitted May 8, 2020; Revised Jun 26, 2020; Accepted Aug 10, 2020
Objective: Temperature could influence protein and amino acid deposition as well as gut microbiota profile and composition. However, the specific effects of ambient temperature on amino acids deposition and gut microbiota composition remain insufficiently understood. Methods: A total of 300 one-day-old Avian broilers were randomly divided into three groups and reared at high, medium, and low temperature (HT, MT, and LT), respectively. Breast muscle and fecal samples were collected for amino acid composition analysis and 16S rRNA gene sequence analysis.

Results: Our data showed that compared to the MT group, there was a decrease of muscle leucine and tyrosine $(\mathrm{p}<0.05)$, as well as an increase of methionine in the HT group $(\mathrm{p}<0.05)$ and a decrease of serine in the LT group. Examination of microbiota shift revealed that at genus level, the relative abundance of Turicibacter and Parabacteroides was increased in the HT group $(\mathrm{p}<0.05)$ and that the relative abundances of Pandoraea, Achromobacter, Prevotella, Brevundimonas, and Stenotrophomonas in the LT group were higher than those in the MT group ( $\mathrm{p}<0.05)$. In addition, there were substantial correlations between microbes and amino acids. In the HT group. Turicibacter was negatively correlated with aspartic acid and tyrosine, whereas Parabacteroides was positively correlated with methionine $(\mathrm{p}<0.05)$. In the LT group, there were multiple positive correlations between Achromobacter and arginine, isoleucine or tyrosine; between Prevotella and cysteine or phenylalanine; between Brevundimonas and cysteine; and between Stenotrophomonas and cysteine as well as a negative correlation between Stenotrophomonas and serine.

Conclusion: Our findings demonstrated that amino acid content of breast muscle and intestinal microbiota profile was affected by different ambient temperatures. Under heat exposure, augmented abundance of Parabacteroides was correlated with elevated methionine. Low temperature treatment may affect muscle tyrosine content through the regulation of Achromobacter.

Keywords: Broiler; Amino Acid Deposition; Fecal Microbiota Composition; Ambient Temperature

\section{INTRODUCTION}

Environment conditions are crucial to poultry welfare and production, as the animals are highly sensitive to temperature-associated environmental challenges [1]. Long-term exposure of poultry to unfavorable environmental temperatures (high or low) can affect the performance of chickens by reduction of body weight gain, impairment of meat quality, induction of excessive immune responses and elevation of mortality [2]. In addition, heat stress or cold stress lead to prominent changes in the gut microbiota composition [3]. The 
animal gastrointestinal tract is a major habitat for numerous species of microbes [4], which play various crucial roles including prevention of the colonization of enteric pathogens through the process of competitive exclusion and the production of bacteriostatic and bactericidal substances [5]. Also, the host metabolism, nutrition, health, and growth performance can be influenced by the enteric microbes via modulating energy balance, nutrient utilization and the development of homeostasis [6].

It has been shown that high or low temperature could change broilers' muscle protein content in broilers $[7,8]$. A previous study showed that amino acid composition of breast muscle was altered under high ambient temperature [9]. Comparison of germ-free mice and conventionalized mice showed that the latter possessed an altered distribution of free amino acids in the gastrointestinal tract and the gut microbiota affected host amino acid metabolism [10]. However, it remains unclear whether intestinal microorganisms exert different effects on amino acid metabolism in breast muscle under different ambient temperatures over a long-term (day 1 to 42). In light of this, the goal of this study was to examine whether long-term temperature stress plays a role in the correlations between amino acid deposition and gut microbiota composition of broilers.

\section{MATERIALS AND METHODS}

\section{Animal ethics statement}

This study was carried out in accordance with the recommendations of the Institutional Animal Care and Use Committee of Yunnan Agricultural University (approval No.: YNAU 20160016), and complied with the guidelines of the institutional administrative committee and ethics committee of laboratory animals.

\section{Experimental design and animal management}

A total of 300 one-day-old avian chicks were purchased from a local supplier (Hunan Shuncheng Industrial Co. Ltd., Hunan, China) and randomly split into three open-circuit calorimetry chambers that were under high temperature (HT), medium temperature (MT), and low temperature (LT), respectively. The temperatures were achieved in a decremental mode in which they began at $36.5^{\circ} \mathrm{C}(\mathrm{HT}), 33.5^{\circ} \mathrm{C}(\mathrm{MT})$, and $30.5^{\circ} \mathrm{C}$ (LT), respectively, and were reduced by $0.5^{\circ} \mathrm{C}$ per day until they reached $22^{\circ} \mathrm{C}, 19^{\circ} \mathrm{C}$, and $16^{\circ} \mathrm{C}$, respectively (Supplementary Table S1). Chicks in each chamber were further subdivided into three areas and provided with a bedding of rice husks, feed and water ad libitum. Apart from the temperature differences, the chickens in all three groups received the same treatments, including diet formulated according to NRC [11] and the same housing (Supplementary Table S2). The chickens were provided with light/dark cycles of $24 / 0$ on D1, 23/1-18/6 from D2 to D8, 12/12 from D9 to D21, 18/6 from D22 to D35, and 18/6-23/1 from D36 to D42 (the light period increased from $18 \mathrm{~h}$ on day 36 to $23 \mathrm{~h}$ on day 42 ); the light intensity was 30 to 60 lux for chickens with a weight of less than $160 \mathrm{~g}$ (about 1 to $8 \mathrm{~d}$ ), and 5 to 10 lux for chickens with a weight above $160 \mathrm{~g}$ (about 9 to $42 \mathrm{~d}$ ). The relative humidity was $30 \%$ to $50 \%$ from D1 to D7, $40 \%$ to $60 \%$ from D8 to D21, and 50\% to $70 \%$ from D22 to D42.

\section{Sample collection}

On day 42, twelve birds (six male and six female) from each group were randomly selected and sacrificed by cervical dislocation. Feces were sampled, preserved in liquid nitrogen, and subjected to DNA extraction and polymerase chain reaction (PCR) amplification. Both sides of the breast muscle were removed and preserved at $-20^{\circ} \mathrm{C}$ for amino acid analysis.

\section{Analysis of muscular amino acids}

For amino acid analysis, the samples preparation was as follows: each breast muscle sample weighted $30 \mathrm{mg}$ was transferred into a hydrolysis tube and digested in $10 \mathrm{~mL}$ of $6 \mathrm{~N} \mathrm{HCl}$ at $110^{\circ} \mathrm{C}$ for $24 \mathrm{~h}$. After filtering, $0.3 \mathrm{~mL}$ of the mixture was transferred into a tube for drying in an oven at $140^{\circ} \mathrm{C}$ for $1 \mathrm{~h}$. Afterwards, $3 \mathrm{~mL}$ sodium citrate buffer solution $(\mathrm{pH} 2.2)$ was used to dissolve the desiccated filtrate sample, followed by the determination of the amino acid composition and content of breast muscle using an amino acid analyzer (Sykam S-433D, Munich, Germany).

\section{DNA extraction and polymerase chain reaction amplification}

Genomic DNA was extracted from fecal samples using the QIAamp Fast DNA Stool Mini Kit (Qiagen, Cat No.19593, Dusseldorf, Germany) according to manufacturer's protocols. The DNA samples were used as the template for PCR. The hypervariable V3-V4 region of the bacteria $16 \mathrm{~S}$ ribosomal RNA genes were amplified using the primer pair 341 F 5'-CCTACGGGRSGCAGCAG)-3' and 806 R 5'-GGAC TACVVGGGTATCTAATC-3' with the following cycling condition: a denaturing step at $95^{\circ} \mathrm{C}$ for $3 \mathrm{~min}$, followed by 30 cycles at $98^{\circ} \mathrm{C}$ for $20 \mathrm{~s}, 58^{\circ} \mathrm{C}$ for $15 \mathrm{~s}$, and $72^{\circ} \mathrm{C}$ for $20 \mathrm{~s}$ and a final extension at $72^{\circ} \mathrm{C}$ for $5 \mathrm{~min}$. The PCR reactions were performed in $30 \mu \mathrm{L}$ mixture containing $15 \mu \mathrm{L}$ of $2 \times \mathrm{KAPA}$ Library Amplification Ready Mix, $1 \mu \mathrm{L}$ of each primer solution $(10 \mu \mathrm{M})$ and $50 \mathrm{ng}$ of template DNA.

\section{Illumina HiSeq PE250 sequencing}

Amplicons were electrophoresed in 2\% agarose gels, followed by extraction from the gel, purification using the AxyPrep DNA Gel Extraction Kit (Axygen Biosciences, Union City, CA, USA) according to the manufacturer's instructions and 
quantified using Qubit 2.0 (Invitrogen, Carlsbad, CA, USA). Libraries were prepared and sequenced on a HiSeq platform (Illumina, Inc., San Diego, CA, USA) for paired-end reads of $250 \mathrm{bp}$, which were overlapping on their 3 ends for concatenation into longer contigs. DNA extraction, library construction and sequencing were performed at Realbio Genomics Institute (Realbio, Shanghai, China). Processing of sequence data was made as previously described [12].

\section{Statistical analysis}

Experimental data including growth performance, amino acid composition of breast muscle and microbial abundances were analyzed using the SPSS 22.0 software (IBM SPSS Statistics for Windows; NY: IBM Corp, Armonk, NY, USA). Shapiro-Wilk test was applied to assess normality. After logarithmic transformation, only data of growth performance and amino acid composition of breast muscle displayed a normal distribution. The general linear model analysis with Duncan multiple comparison test was used for parametric data and Kruskal-Wallis analysis of variance performed on ranks was used for the microbial abundances at phylum and genus level. The growth performance and amino acid data were expressed as the mean, and the pooled standard error of mean was provided. To assess the correlation between dominant genera and amino acids, the Spearman's test in GraphPad Prism 7.0 was performed, and $\mathrm{p}<0.05$ was considered as the criterion for statistical significance.

\section{RESULTS}

\section{Growth performance}

The three temperature treatments resulted in detectable differences in the growth performance of the broilers. On day 42 , the final body weight, average daily gain and breast muscle weight of broilers in the HT and MT groups were significantly higher $(\mathrm{p}<0.05)$ than that in the LT group. The average daily feed intake was 98.31, 97.03, and 91.1 for HT, MT, and LT groups, respectively. The ratio of feed gain of broilers in the LT (2.18) group was higher than that in the HT (1.98) group $(\mathrm{p}<0.05)$ and both groups were higher than that in the MT (1.87) group $(\mathrm{p}<0.05)($ Table 1$)$.

\section{Amino acid deposition and crude protein analysis}

We next examined the influences of the three temperature treatments on amino acid deposition and crude protein (CP) content in the breast muscle. As shown in Table 2, CP content in the LT group was higher than that in the MT and HT groups $(\mathrm{p}<0.05)$. The content of serine in breast muscle of MT group was significantly higher $(\mathrm{p}<0.05)$ than that of LT group. Lower methionine of breast muscle in MT group was observed compared with the HT and LT groups ( $\mathrm{p}<0.05)$. Compared with the MT group, the broilers in the HT group had less leucine $(\mathrm{p}<0.05)$, and the HT chicks had lower tyrosine than the MT and LT chicks ( $\mathrm{p}<0.05)$.

\section{Sequence and data analysis}

Analysis of 16S rRNA gene amplicons generated 2,124,545 clean reads from the 36 fecal samples, resulting in 37,479 to 64,768 reads per sample with an average length of $416 \mathrm{bp}$. To avoid the effect of the sequence depth on the measurement of microbial composition, we rarefied the library size to 32,296 reads per sample using the rarefy function in QIIME pipeline (Supplementary Table S3). With a 97\% sequence similarity, 385 core operational taxonomic units (OTUs) were generated. As a consequence, and the OTUs numbers were 540, 709, or 956 for the HT, MT, or LT groups, and the proportion of core taxa in the feces of broilers was $71 \%, 54 \%$, or $40 \%$, respectively (Figure 1).

The microbial diversity indices were calculated based on the OTUs of each library. And the Chaol index, phylogenetic diversity (PD) whole tree index, good's coverage, Shannon index, observed species index, and Simpson index (Supplementary Table S4). The results of alpha diversity analysis is shown in Figure 2, showing that the indices of chao1 and PD whole tree in the MT and LT groups were significantly higher $(\mathrm{p}<0.05)$ than those in the HT group, and that the index of good's coverage in the HT group was significantly higher $(\mathrm{p}<0.05)$ than those in the MT and LT groups.

Table 1. Growth performance of broilers in different environmental temperatures

\begin{tabular}{|c|c|c|c|c|c|}
\hline Group ID & $\mathrm{HT}^{1)}$ & $\mathrm{MT}^{1)}$ & $\mathrm{LT}^{1)}$ & SEM & p-value \\
\hline FBW (g) & $2,141.50^{a}$ & $2,225.00^{a}$ & $1,801.00^{b}$ & 43.16 & $<0.001$ \\
\hline ADFI (g) & 98.31 & 97.03 & 91.1 & 1.49 & 0.108 \\
\hline ADG (g) & $49.89^{\mathrm{a}}$ & $51.89^{\mathrm{a}}$ & $41.79^{b}$ & 1.03 & $<0.001$ \\
\hline$F / G$ & $1.98^{b}$ & $1.87^{c}$ & $2.18^{\mathrm{a}}$ & 0.023 & $<0.001$ \\
\hline Breast muscle weight (g) & $374.50^{\mathrm{a}}$ & $386.50^{\mathrm{a}}$ & $321.50^{b}$ & 9.56 & 0.008 \\
\hline
\end{tabular}

Values reported as means $(n=12)$.

SEM, standard error of means for 12 broilers each; FBW, final body weight; ADFI, average daily feed intake; ADG, average daily gain; F/G, the ratio of feed gain.

${ }^{1)} H T$, high temperature; $M T$, medium temperature; $L T$, low temperature.

${ }_{a, b}$ Means in the same row with different superscripts differ statistically $(p<0.05)$. 
Table 2. Effects of different environmental temperatures on crude protein and amino acid composition in broilers breast muscle

\begin{tabular}{|c|c|c|c|c|c|}
\hline Group ID & $\mathrm{HT}^{1)}$ & $\mathrm{MT}^{1)}$ & $\mathrm{LT}^{1)}$ & SEM & $\mathrm{p}$-value \\
\hline Alanine & 1.34 & 1.37 & 1.32 & 0.013 & 0.602 \\
\hline Glycine & $1.04^{\mathrm{ab}}$ & $1.12^{\mathrm{a}}$ & $1.04^{b}$ & 0.012 & 0.061 \\
\hline Proline & 0.85 & 0.86 & 0.82 & 0.0082 & 0.446 \\
\hline Serine & $0.70^{\mathrm{ab}}$ & $0.8^{a}$ & $0.67^{b}$ & 0.0088 & 0.027 \\
\hline Arginine & 1.53 & 1.6 & 1.65 & 0.016 & 0.205 \\
\hline Histidine & $0.70^{\mathrm{b}}$ & $0.78^{a}$ & $0.75^{\mathrm{ab}}$ & 0.016 & 0.052 \\
\hline Isoleucine & 1.25 & 1.25 & 1.38 & 0.011 & 0.27 \\
\hline Leucine & $2.14^{b}$ & $2.26^{a}$ & $2.24^{\mathrm{ab}}$ & 0.019 & 0.039 \\
\hline Lysine & 2.33 & 2.45 & 2.47 & 0.024 & 0.307 \\
\hline Valine & 1.27 & 1.28 & 1.24 & 0.013 & 0.737 \\
\hline Phenylalanine & 1.18 & 1.21 & 1.27 & 0.011 & 0.149 \\
\hline Tyrosine & $0.85^{b}$ & $0.95^{\mathrm{a}}$ & $0.91^{\mathrm{a}}$ & 0.0095 & 0.001 \\
\hline Aspartic acid & 2.15 & 2.21 & 2.17 & 0.21 & 0.661 \\
\hline Glutamic acid & 3.47 & 3.53 & 3.42 & 0.034 & 0.661 \\
\hline Cysteine & 0.23 & 0.28 & 0.27 & 0.0037 & 0.284 \\
\hline Methionine & $0.70^{a}$ & $0.47^{b}$ & $0.62^{a}$ & 0.011 & 0.001 \\
\hline Threonine & 0.85 & 0.86 & 0.83 & 0.011 & 0.776 \\
\hline Total AA & 22.62 & 23.28 & 22.99 & 0.22 & 0.594 \\
\hline Crude protein & $23.91^{\mathrm{b}}$ & $24.07^{b}$ & $25.69^{a}$ & 0.23 & $<0.001$ \\
\hline
\end{tabular}

Values reported as means $(n=12)$.

SEM, standard error of means for 12 broilers each; AA, amino acid.

1) HT, high temperature; MT, medium temperature; LT, low temperature.

$a, b$ Means in the same row with different superscripts differ statistically $(p<0.05)$.

\section{Comparison of gut bacterial composition at phylum level based on $16 \mathrm{~S}$ rRNA amplicon sequence}

There were 8,16 , and 14 phyla being identified within the complete dataset in the HT, MT, and LT groups, respectively (Supplementary Figure S1). Firmicutes, Bacteroidetes and

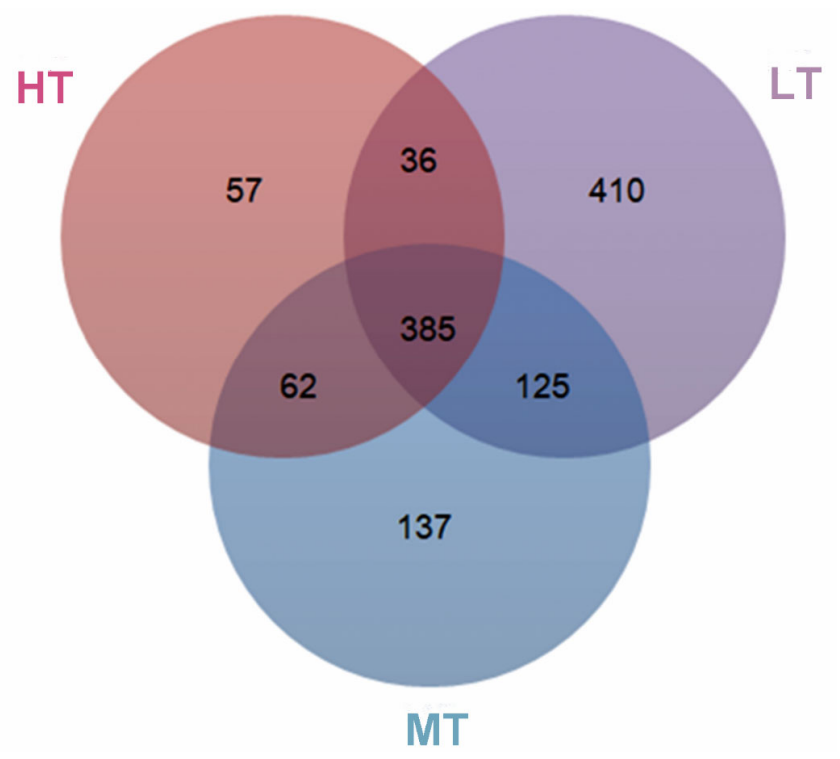

Figure 1. Venn diagram of OTUs clustered at $97 \%$ sequence identity across $H T, M T$, and LT groups. The different colors respectively represent HT, MT, LT groups, and the overlapping area represent shared OTUs numbers between different groups. OUTs, operational taxonomic unit; $\mathrm{HT}$, high temperature; MT, medium temperature; LT, low temperature.
Proteobacteria were the only three phyla shared among the three groups, with each accounting for $\geq 10 \%$ of the fecal microbiota. Overall, the relative abundance of the three major phyla was comparable among the three groups. Specifically, the proportions of Firmicutes were $60.56 \%$ in the HT group, $57.62 \%$ in the MT group and $53.92 \%$ in the LT group; that of Bacteroidetes were $22.48 \%$ in the HT group, $28.98 \%$ in the MT group and $21.91 \%$ in the LT group; that of Proteobacteria were $15.95 \%$ in the HT group, $12.22 \%$ in the MT group and $22.76 \%$ in the LT group, respectively (Figure 3 ). The other bacterial phyla had relative abundances lower than $1 \%$ in all groups at varying magnitudes.

Comparison of gut bacterial composition at genus level based on 16S rRNA amplicon sequence

At the genus level, the top 20 abundant genera for all three groups were ClostridiumXI, Bacteroides, Escherichia/Shigella, Barnesiella, Enterococcus, Lactobacillus, Pandoraea, Alistipes, Turicibacter, Clostridium XIVa, Faecalibacterium, Achromobacter, Butyricicoccus, Oscillibacter, Variovorax, Clostridium IV, Clostridium XIVb, Clostridium sensu strict, Phascolarctobacterium, and Parabacteroides in the three groups. Among these, the major taxa included Clostridium XI ( $8.77 \%$ in HT, $17.76 \%$ in MT, and $25.68 \%$ in LT), Bacteroides (11.29\% in HT, $11.24 \%$ in MT, and $13.06 \%$ in LT), Escherichia/Shigella (15.84\% in HT, $11.36 \%$ in MT, and $8.31 \%$ in LT) and Barnesiella $(7.82 \%$ in HT, $15.80 \%$ in MT, and 5.07\% in LT) (Supplementary Figure S2). 

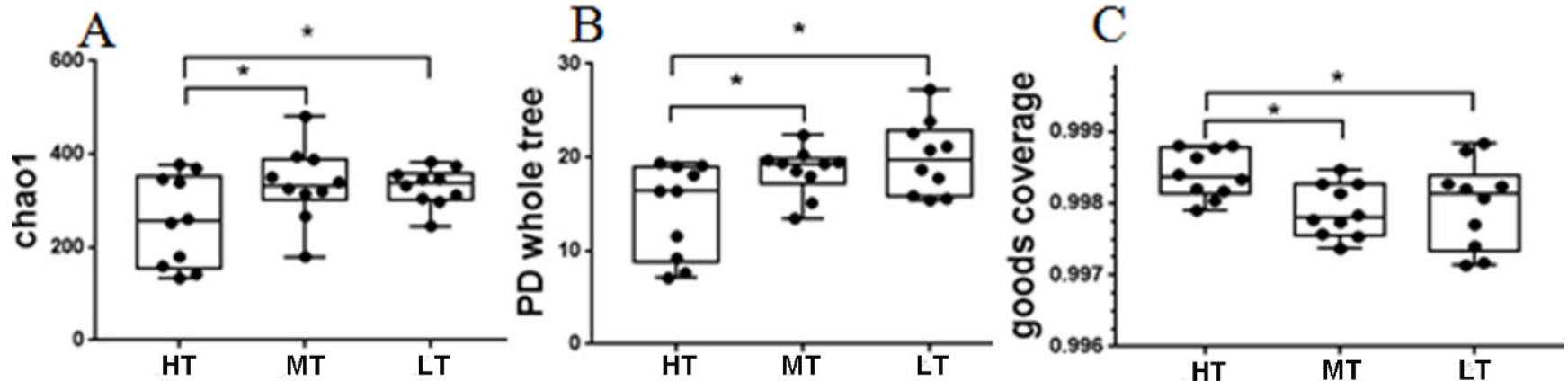

$\mathrm{D}$

E

$\mathrm{F}$
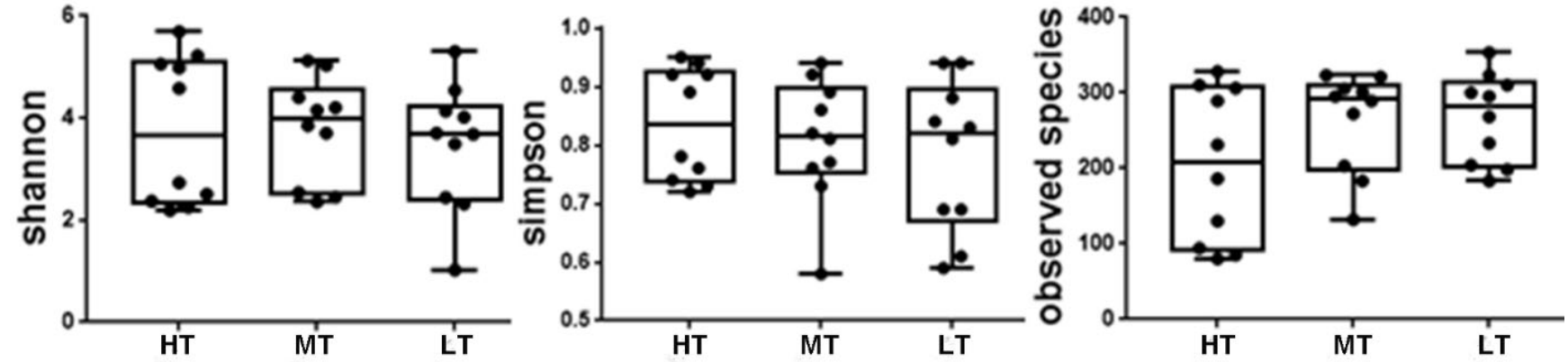

Figure 2. Diversity estimation of the $16 \mathrm{~S}$ rRNA gene libraries of the broilers' feces. The boxes represent interquartile range (IQR), and the line between boxes indicate median value. ${ }^{*} \mathrm{p}<0.05$. HT, high temperature; MT, medium temperature; LT, low temperature.

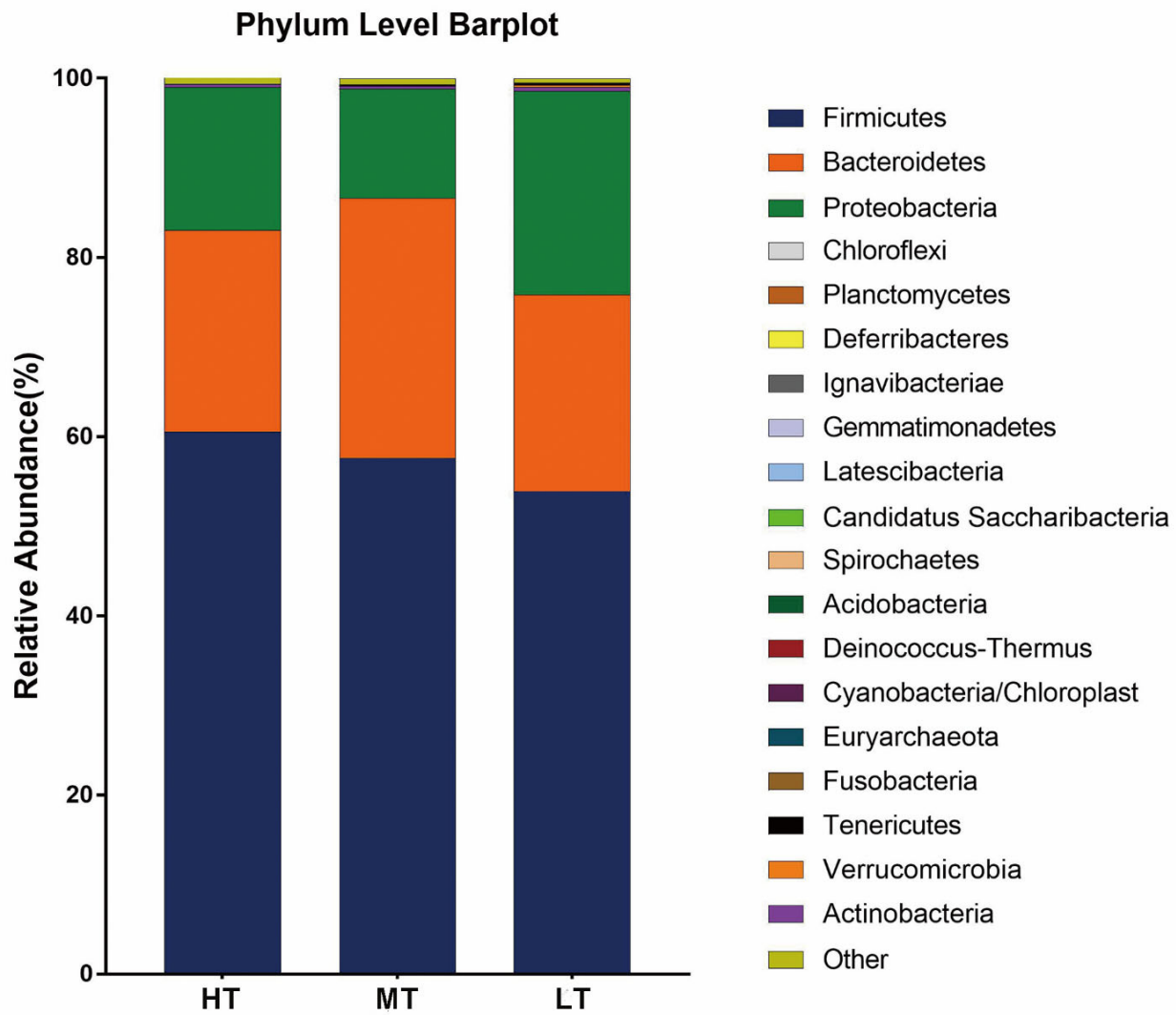

Figure 3. Composition of the dominant microbiome at phylum level. The composition of each sample is based on the ribosomal database project (RDP) taxonomic assignment of the $16 \mathrm{~S}$ rDNA sequences. HT, high temperature; MT, medium temperature; LT, low temperature. 


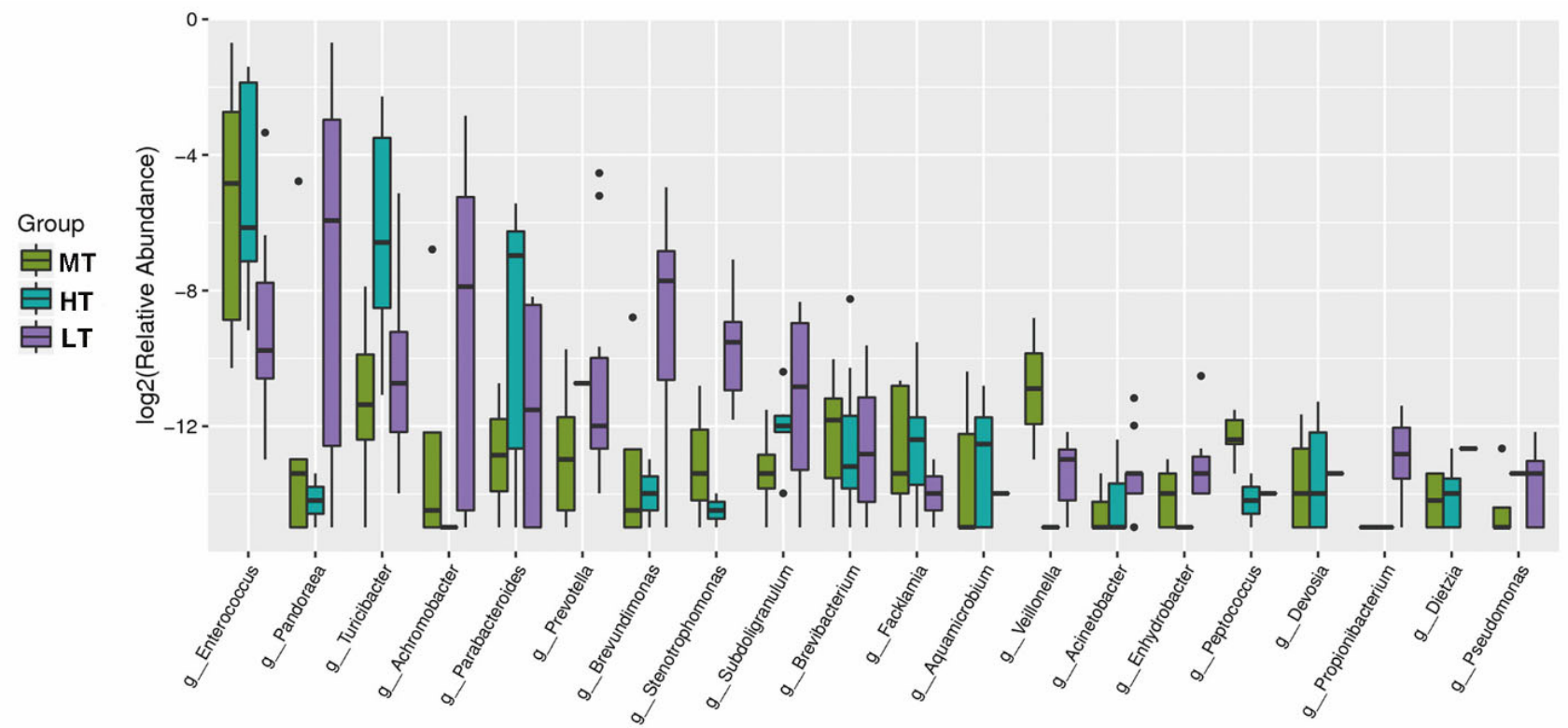

Figure 4. Boxplot of the top 20 most differentially abundant genus in the HT, MT and LT groups. The value of $\log _{2}$ (relative abundance) was used in the plot. The dots in the figure represent deviation values. HT, high temperature; MT, medium temperature; LT, low temperature.

The relative abundances of Turicibacter (4.88\%), Parabacteroides (0.73\%), Brevibacterium (0.043\%), Facklamia (0.028\%), Aquamicrobium (0.016\%) or Dietzia (0.0021\%) were higher in the HT group compared with that in the MT group $(\mathrm{p}<0.05)$. In addition, the relative abundance of $E n$ terococcus in the MT (13\%) and HT (12\%) groups was higher

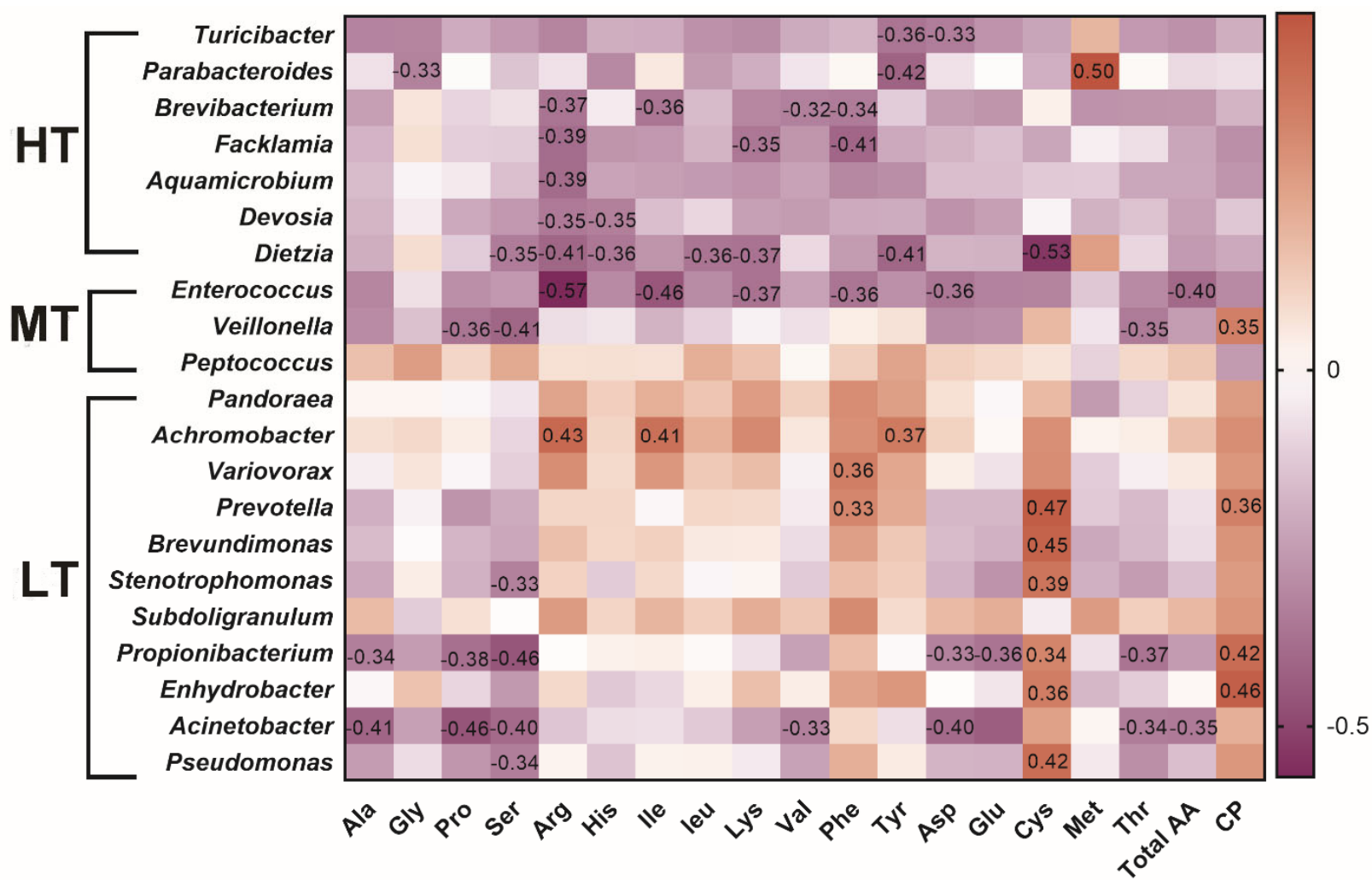

Figure 5. Heatmap of the correlation between microbial changes and amino acids. Spearman's test was used to calculate correlation coefficient The negative correlation was expressed by purple color, and the positive correlation was expressed by red color. HT, high temperature; MT, medium temperature; LT, low temperature. 
than that in the LT (1\%) group ( $<<0.05)$; Veillonella in MT group was higher than in HT and LT groups $(\mathrm{p}<0.05)$. The abundance of Pandoraea (9.9\%), Achromobacter (2.14\%), Prevotella (0.61\%), Brevundimonas (0.55\%), Stenotrophomonas (0.12\%), Subdoligranulum (0.092\%), Enhydrobacter (0.011\%), Acinetobacter (0.01\%), and Propionibacterium $(0.011 \%)$ in the LT group were significantly higher than those in HT and MT groups ( $<<0.05)$ (Figure 4). The results indicated that the different temperature treatments led to substantial shift of gut microbiota.

\section{Correlations between microbes and amino acid deposition}

As shown in Figure 5, positive correlation was revealed between the protein content and Veillonella. In the HT group, Turicibacter was negatively correlated with aspartic acid ( $\mathrm{p}=$ $0.044, \mathrm{R}=-0.33)$ and tyrosine $(\mathrm{p}=0.030, \mathrm{R}=-0.36)$; Parabacteroides was negatively associated with glycine or tyrosine and positively correlated with methionine $(\mathrm{p}=0.0019, \mathrm{R}=$ $0.50)$; Dietzia was negatively correlated with leucine $(\mathrm{p}=0.030$, $\mathrm{R}=-0.36$ ). In the MT group, negative correlation were detected between Enterococcus and arginine $(\mathrm{p}=0.00028, \mathrm{R}=$ $-0.57)$ or isoleucine $(\mathrm{p}=0.0051, \mathrm{R}=-0.46)$ and between Veillonella and serine $(\mathrm{p}=0.013, \mathrm{R}=-0.40)$, threonine $(\mathrm{p}=0.038$, $\mathrm{R}=-0.35)$, or proline $(\mathrm{p}=0.030, \mathrm{R}=-0.36)$. In the LT group, associations were identified between Achromobacter and arginine $(\mathrm{p}=0.0083, \mathrm{R}=0.43)$, isoleucine $(\mathrm{p}=0.014, \mathrm{R}=0.41)$, between Variovorax and phenylalanine $(\mathrm{p}=0.03, \mathrm{R}=0.36)$, between Prevotella and cysteine $(\mathrm{p}=0.0034, \mathrm{R}=0.47)$, or phenylalanine ( $\mathrm{p}=0.046, \mathrm{R}=0.33$ ), between Brevundimonas, and cysteine $(\mathrm{p}=0.0053, \mathrm{R}=0.45)$, between Stenotrophomonas and serine ( $\mathrm{p}=0.048, \mathrm{R}=-0.33)$, or cysteine $(\mathrm{p}=0.018, \mathrm{R}=$ 0.39) (Supplementary Table S5, Table S6). The results indicated that gut microbiota composition influences amino acid composition.

\section{DISCUSSION}

Environment is important factor affecting the performance of modern poultry production [13]. Birds are homeotherms and can maintain their body temperature within a narrow range. However, when the body temperature substantially deviates from the normal range resulting from exposure to unfavorable environmental conditions, a cascade of irreversible thermoregulatory events may occur, which are detrimental and even lethal to the birds [14]. Studies have shown that long-term exposure to cold environment leads to a decrease in protein synthesis in rats [15] and calves [16]. Differences in composition of amino acids, especially those essential amino acids, can greatly influence the protein synthesis. A previous study reported that after 14 days ( $28 \mathrm{~d}$ to $42 \mathrm{~d}$ ) of heat exposure (environment temperature was $32^{\circ} \mathrm{C}$ ), muscle amino acid concentration was reduced of broilers [17]. The detrimental effect on muscular amino acids was also observed in rats exposed to cold stress at $4^{\circ} \mathrm{C}$ [18]. These results are consistent with our results to collectively show that heat and cold stresses influence the content of muscular crude protein and amino acid composition.

In the present study, the contents of serine and glycine were significantly decreased by long-term cold stress, as were those of leucine, histidine and tyrosine by heat treatment in broilers. The methionine levels in HT and LT groups were significantly higher than that in MT group. Studies have revealed that exposure to extreme temperatures led to oxidative stress [19]. As an essential amino acid in chickens, methionine has a positive effect on the expression of stress related genes and thus helps to protect cells from oxidative challenge [20]. It is plausible that under extreme environment, the broilers require a higher methionine concentration. Therefore, temperature is one of the main factors affecting amino acid metabolism in broilers.

In healthy chickens, the composition of intestinal microbiota remains relatively stable, which may be disrupted by various factors such as pathogen invasion, antibiotic administration and environmental stress (e.g., extremely high or low temperature, overcrowding, poor feeding, transportation) [21]. Fecal microbiome analyses suggested bacterial richness was a major marker for gut health [22]. And Chao1 indices are usually used to estimate bacterial richness. In this study, Chaol showed that the gut microbiota in MT and LT groups were more abundant than that in HT group.

It has been shown that intestinal microflora could be considered as an important indicator of intestinal health [23]. The composition and relative abundance of intestinal microbiota are influenced by environmental temperature [24]. Enterococcus is a large genus of lactic acid bacteria in the phylum Firmicutes [25]. Although some species of Enterococcus are potentially pathogenic, their toxicity is generally low and they are natural commensals in human and animal gastrointestinal tracts [26]. Our study showed that the proportion of Enterococcus in the MT and HT groups was higher than that in the LT group, indicating their abundance was affected by heat stress. Barnesiella is a genus in the family of Porphyromonadaceae and is a major genus detected in mouse intestines. It is associated with hyperglycemia, insulin resistance, hepatic steatosis and inflammation in rodents [27]. Barnesiella is also one of the most abundant genera in human feces and can be used to treat vancomycin-resistant Streptococcus faecalis colonization [28]. In this study, Barnesiella was a predominant in all samples and its relative abundance in the MT group was higher than that of the other two groups. The results indicated Barnesiella was also one of the most abundant genera in broilers and its abundance was influenced by both heat and cold stresses. 
Yang et al (2021) Anim Biosci 34:93-101

The resident species of the gut microbiota can alter the bioavailability of amino acids by utilizing of several amino acids that are originated from both alimentary and endogenous proteins [29]. Additionally, the gut microbiota can synthesize amino acids and provide them to the host [30]. Therefore, the gut microbiota is a potential regulatory factor in amino acid homeostasis which may affect the muscular amino acids deposition. In the present study, Parabacteroides was augmented in the feces of the HT-treated broilers. The correlation analysis revealed that in the HT group, this genus was negatively associated with glycine or tyrosine and positively correlated with methionine. The results suggested that under heat exposure, augmenting the abundance of Parabacteroides correlated with elevated concentration of methionine. In addition, Achromobacter was enriched in the feces of the LT group, and Achromobacter was positively correlated with tyrosine in the MT and LT groups, suggesting that low temperature treatment may affect tyrosine through the regulation of Achromobacter. Moreover, our analyses revealed that Stenotrophomonas was negatively correlated with serine and that the serine content in the LT group was lower than that in the MT group, suggesting that low temperature treatment may affect serine metabolism and that it might involve Stenotrophomonas.

$16 \mathrm{~S}$ rRNA gene-based analysis revealed that whereas the proportion of the three major phyla, namely Firmicutes, Bacteroidetes and Proteobacteria, were comparable among the three groups, there were substantial differences at genus level. Specifically, significant differences of multiple genera (eg., Turicibacter, Parabacteroides, Enterococcus, Pandoraea, Achromobacter, Variovorax, Prevotella, Brevundimonas, and Stenotrophomonas) were found in all three groups. Furthermore, our findings suggested some connections between gut microbiota and muscular amino acid composition. Our findings provide new evidence for better understanding the involvement of gut microbiota in regulation of amino acid metabolism and how the process is affected by ambient temperatures. Furthermore, our study also provides crucial experimental basis for developing microbiota-based supplements to improve meat quality in broilers.

\section{CONFLICT OF INTEREST}

We certify that there is no conflict of interest with any financial organization regarding the material discussed in the manuscript.

\section{ACKNOWLEDGMENTS}

The authors want to thank Qiaoping Ji for providing the poultry feed. We thank Qihua Li for experimental equipment. This study was supported by the National Key R\&D
Program of China (2016YFD0500501).

\section{REFERENCES}

1. Lara LJ, Rostagno MH. Impact of heat stress on poultry production. Animals 2013;3:356-69. https://doi.org/10.3390/ ani3020356

2. He X, Lu Z, Ma B, et al. Effects of chronic heat exposure on growth performance, intestinal epithelial histology, appetiterelated hormones and genes expression in broilers. J Sci Food Agric 2018;98:4471-8. https://doi.org/10.1002/jsfa.8971

3. Burkholder KM, Thompson KL, Einstein ME, Applegate TJ, Patterson JA. Influence of stressors on normal intestinal microbiota, intestinal morphology, and susceptibility to Salmonella enteritidis colonization in broilers. Poult Sci 2008;87:1734-41. https://doi.org/10.3382/ps.2008-00107

4. Nicholson JK, Holmes E, Kinross J, et al. Host-gut microbiota metabolic interactions. Science 2012;336:1262-7. https://doi. org/10.1126/science.1223813

5. Clavijo V, Flórez MJV. The gastrointestinal microbiome and its association with the control of pathogens in broiler chicken production: a review. Poult Sci 2018;97:1006-21. https://doi. org/10.3382/ps/pex359

6. Koren O, Goodrich JK, Cullender TC, et al. Host remodeling of the gut microbiome and metabolic changes during pregnancy. Cell 2012;150:470-80. https://doi.org/10.1016/j.cell. 2012.07.008

7. Zhang ZY, Jia GQ, Zuo JJ, et al. Effects of constant and cyclic heat stress on muscle metabolism and meat quality of broiler breast fillet and thigh meat. Poult Sci 2012;91:2931-7. https:// doi.org/10.3382/ps.2012-02255

8. Akşit M, Yalçin S, Ozkan S, Metin K, Ozdemir D. Effects of temperature during rearing and crating on stress parameters and meat quality of broilers. Poult Sci 2006;85:1867-74. https:// doi.org/10.1093/ps/85.11.1867

9. Ito K, Erwan E, Nagasawa M, Furuse M, Chowdhury VS. Changes in free amino acid concentrations in the blood, brain and muscle of heat-exposed chicks. Br Poult Sci 2014; 55:644-52. https://doi.org/10.1080/00071668.2014.957653

10. Mardinoglu A, Shoaie S, Bergentall M, et al. The gut microbiota modulates host amino acid and glutathione metabolism in mice. Mol Syst Biol 2015;11:834. https://doi.org/10.15252/ msb. 20156487

11.Committee on Nutrient Requirements of Poultry, National Research Council. Nutrient requirements of poultry. 9th revised ed. Washington, DC, USA: National Academies Press; 1994.

12.Li X, Cao Z, Yang Y, et al. Correlation between jejunal microbial diversity and muscle fatty acids deposition in broilers reared at different ambient temperatures. Sci Rep 2019;9:11022. https://doi.org/10.1038/s41598-019-47323-0

13. Alleman F, Leclercq B. Effect of dietary protein and environ- 
mental temperature on growth performance and water consumption of male broiler chickens. Br Poult Sci 1997;38:60710. https://doi.org/10.1080/00071669708418044

14. Kamel NN, Ahmed AMH, Mehaisen GMK, Mashaly MM, Abass AO. Depression of leukocyte protein synthesis, immune function and growth performance induced by high environmental temperature in broiler chickens. Int J Biometeorol 2017;61:1637-45. https://doi.org/10.1007/s00484-017-1342-0

15. Samuels SE, Thompson JR, Christopherson RJ. Skeletal and cardiac muscle protein turnover during short-term cold exposure and rewarming in young rats. Am J Physiol Regul Integr Comp Physiol 1996;270:R1231-9. https://doi.org/10. 1152/ajpregu.1996.270.6.R1231

16. Scott SL, Christopherson RJ, Thompson JR, Baracos VE. The effect of a cold environment on protein and energy metabolism in calves. Br J Nutr 1993;69:127-39. https://doi.org/10.1079/ BJN19930015

17. Ma B, He X, Lu Z, et al. Chronic heat stress affects muscle hypertrophy, muscle protein synthesis and uptake of amino acid in broilers via insulin like growth factor-mammalian target of rapamycin signal pathway. Poult Sci 2018;97:41508. https://doi.org/10.3382/ps/pey291

18.Smith OL, Huszar G, Davidson SB, Davis E. Effects of acute cold exposure on muscle amino acid and protein in rats. J Appl Physiol 1982;52:1250-6. https://doi.org/10.1152/jappl. 1982.52.5.1250

19.Lin H, Decuypere E, Buyse J. Acute heat stress induces oxidative stress in broiler chickens. Comp Biochem Physiol A Mol Integr Physiol 2006;144:11-7. https://doi.org/10.1016/ j.cbpa.2006.01.032

20.Luo S, Levine RL. Methionine in proteins defends against oxidative stress. FASEB J 2009;23:464-72. https://doi.org/ 10.1096/f.08-118414

21.Lan PTN, Sakamoto M, Benno Y. Effects of two probiotic Lactobacillus strains on jejunal and cecal microbiota of broiler chicken under acute heat stress condition as revealed by molecular analysis of $16 \mathrm{~S}$ rRNA genes. Microbiol Immunol 2004;48:917-29. https://doi.org/10.1111/j.1348-0421.2004. tb03620.x

22. Lozupone CA, Stombaugh JI, Gordon JI, Jansson JK, Knight R. Diversity, stability and resilience of the human gut microbiota. Nature 2012;489:220-30. https://doi.org/10.1038/nature 11550

23.Xu ZR, Hu CH, Xia MS, Zhan XA, Wang MQ. Effects of dietary fructooligosaccharide on digestive enzyme activities, intestinal microflora and morphology of male broilers. Poult Sci 2003;82:1030-6. https://doi.org/10.1093/ps/82.6.1030

24. Wang XJ, Feng JH, Zhang MH, Li XM, Ma DD, Chang SS. Effects of high ambient temperature on the community structure and composition of ileal microbiome of broilers. Poult Sci 2018;97:2153-8. https://doi.org/10.3382/ps/pey032

25.Torres C, Alonso CA, Ruiz-Ripa L, León-Sampedro R, Del Campo R, Coque TM. Antimicrobial resistance in Enterococcus spp. of animal origin. Microbiol Spectr 2018;6:185-227. https:// doi.org/10.1128/microbiolspec.ARBA-0032-2018

26. Arias CA, Murray BE. The rise of the Enterococcus: beyond vancomycin resistance. Nat Rev Microbiol 2012;10:266-78. https://doi.org/10.1038/nrmicro2761

27.Le Roy T, Llopis M, Lepage P, et al. Intestinal microbiota determines development of non-alcoholic fatty liver disease in mice. Gut 2013;62:1787-94. https://doi.org/10.1136/gutjnl2012-303816

28. Wylie KM, Truty RM, Sharpton TJ, et al. Novel bacterial taxa in the human microbiome. PLoS One 2012;7:e35294. https:// doi.org/10.1371/journal.pone.0035294

29. Neis EPJG, Dejong CH, Rensen SS. The role of microbial amino acid metabolism in host metabolism. Nutrients 2015;7:2930-46. https://doi.org/10.3390/nu7042930

30.Lin R, Liu W, Piao M, Zhu H. A review of the relationship between the gut microbiota and amino acid metabolism. Amino Acids 2017;49:2083-90. https://doi.org/10.1007/ s00726-017-2493-3 